\title{
Degradation and miscibility of poly(DL-lactic acid)/poly(glycolic acid) composite films: Effect of poly(DL-lactic-co-glycolic acid)
}

\author{
ZHIGANG MA, NA ZHAO* and CHENGDONG XIONG ${ }^{\dagger}$ \\ School of Chemical and Material Engineering, Huangshi Institute of Technology, Huangshi, 435003, P. R. China \\ ${ }^{\dagger}$ Chengdu Organic Chemicals Co. Ltd., Chinese Academy of Sciences, Chengdu 610041, P. R. China
}

MS received 29 November 2010; revised 4 October 2011

\begin{abstract}
The in vitro degradation behaviour of poly(glycolic acid) (PGA) and its composite films containing poly(DL-lactic acid) (PDLLA) and poly(DL-lactic-co-glycolic acid) (PDLGA) were investigated via mass loss, scanning electron microscopy (SEM) and differential scanning calorimetry (DSC). All the films were prepared by solution casting, using 1,1,1,3,3,3-hexafluoro-2-propanol (HFIP) as the solvent. Since the degradation rate of PDLLA is lower than that of PGA, those of the PDLLA/PGA composite films decreased. As a compatibilizer, PDLGA improved the compatibility and hydrolytic stability of PDLLA/PGA composite films. Changes in the composite films indicate that this kind of PGA-based composite biomaterial may be applicable to device design for clinical application in the future.
\end{abstract}

Keywords. Polymers; composite materials; thin films; microstructure; compatibility.

\section{Introduction}

Poly(glycolic acid) (PGA), poly(DL-lactic acid) (PDLLA) and poly(DL-lactic-co-glycolic acid) (PDLGA) have been widely studied and applied in biomedical fields because of their good biodegradability, biocompatibility and reasonably good mechanical properties (Doi et al 2004). However, due to high hydrophilicity, PGA degrades very rapidly (Choueka et al 1995), limiting its clinical applications. To overcome this, it is necessary to modify the degradation rates of PGA.

Previous research reveals that blending PGA with PLA might be effective in decreasing the degradation rate of PGA (You et al 2005). However, our study revealed that PDLLA and PGA were not very compatible. To remedy this, we selected PDLGA as the compatibilizer. In this study, PDLGA/PDLLA/PGA composite films with different ratios were prepared, and their preliminary degradation behaviour and morphology were investigated. We aimed to achieve some success in modifying the degradation rate of PGA.

\section{Experimental}

\subsection{Materials}

PGA (melt volume index $=10 \mathrm{~cm}^{3} / 10 \mathrm{~min}$ ) were purchased from Purac $\mathrm{Co}$ and quenched to make it more dissolvable. PDLLA (weight-average molecular weight $=1.16 \times 10^{6}$, polydispersity $=3.05$ ) and PDLGA (weight-average molecular weight $=2.72 \times 10^{5}$, polydispersity $=1.98$, DL-lactic acid and glycolic acid are in 50:50 molar proportions) were

\footnotetext{
*Author for correspondence (zhaona014112034@163.com)
}

purchased from DIKANG Biomedical Co. HFIP and isopropanol were purchased from DuPont and Chengdu Kelong Chemical Co. respectively.

\subsection{PDLLA/PGA and PDLGA/PDLLA/PGA blend solution}

The weighed quantity of PGA, PDLLA and PDLGA were dissolved in HFIP according to predetermined ratios $(10 \%$, $20 \%, 30 \%$ and $40 \%$ PDLLA/PGA, $1 \%, 2 \%, 5 \%$ and $10 \%$ PDLGA 30\% PDLLA/PGA, wt $\%$ ). The polymer concentrations of all the solutions were $5 \mathrm{wt} \%$.

\subsection{Solution casting}

The blend solution was poured into a glass mould and the solvent was evaporated quickly in air at room temperature for $1 \mathrm{~h}$ to prevent the degradation of PGA. The films were then washed by isopropanol for 3 times, then placed in a vacuum oven at ambient temperature for 2 weeks to evaporate any remaining solvent, leaving behind dried films with a thickness of about $0 \cdot 15 \mathrm{~mm}$.

\subsection{In vitro degradation}

All the films were cut into a rectangular shape with dimensions of $20 \times 10 \times 0.15 \mathrm{~mm}^{3}$, immersed in $5 \mathrm{~mL}$ phosphate buffer solution (PBS, pH 7.4) at $37^{\circ} \mathrm{C}$ after being weighed (initial dry mass, $m_{\mathrm{i}}$ ). Degradation media were not renewed during the degradation period. After each degradation period, the samples were removed from the media and washed thoroughly with water, dried in a vacuum oven at 
ambient temperature for 2 weeks and then weighed (dry mass after degradation, $m_{\mathrm{d}}$ ).

\subsection{Characterization}

Morphologies of all the films were observed under a JEOL scanning electron microscope (SEM) (Model JSM-6300) after gold coating.

Differential scanning calorimetry (DSC) was performed using a TA DSC (Model Q20). To obtain the cooling thermogram, samples were heated to $250^{\circ} \mathrm{C}$ to remove thermal history and then cooled at a cooling rate of $10^{\circ} \mathrm{C} / \mathrm{min}$ in flushing nitrogen.

Crystallization enthalpy $\left(\Delta H_{\mathrm{c}}, \mathrm{J} / \mathrm{g}\right)$ can be determined from DSC thermograms. The crystallinity $\left(x_{\mathrm{c}}\right)$ was calculated using (1), where $139 \mathrm{~J} / \mathrm{g}$ is the enthalpy of fusion of infinite PGA crystals reported by Montes de Oca and Ward (2006):

$$
x_{\mathrm{c}}(\%)=100 \times \Delta H_{\mathrm{c}} / 139 .
$$

\section{Results and discussion}

\subsection{Mass loss}

Mass loss was monitored throughout the degradation and was defined using (2), where $m_{\mathrm{i}}$ was the initial dry mass and $m_{\mathrm{d}}$ the dry mass after degradation.

$$
\text { Mass loss }=\frac{\left(m_{\mathrm{i}}-m_{\mathrm{d}}\right)}{m_{\mathrm{i}}} \times 100 \% .
$$

Figure 1 shows changes in the mass loss of PDLLA/PGA composite films with varying PDLLA contents after $5 \mathrm{~h}$ of degradation. It is evident that the pure PGA film has lost

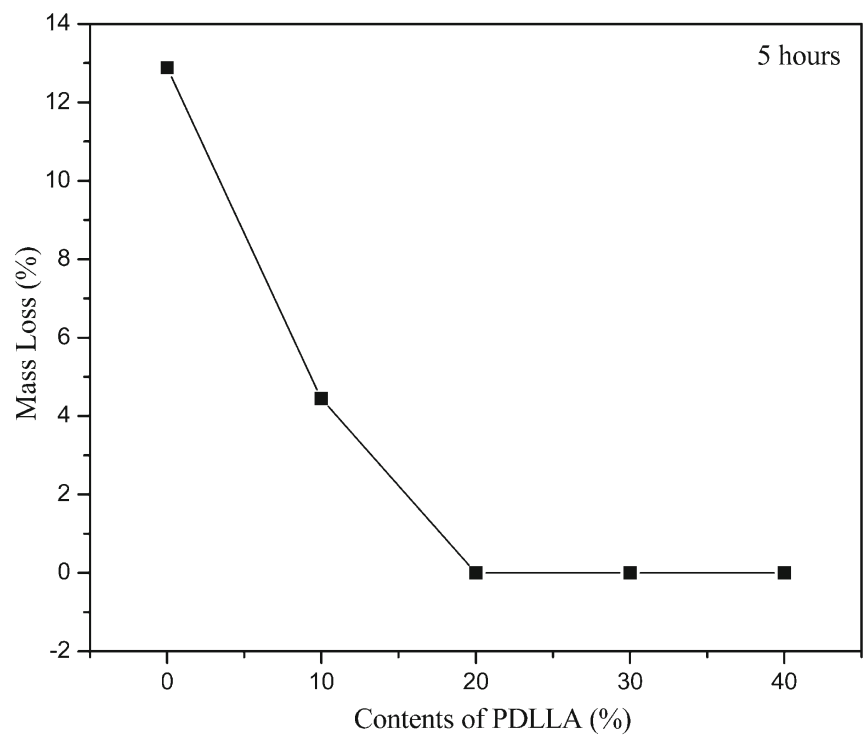

Figure 1. Mass loss of PDLLA/PGA composite films with varying PDLLA contents after $5 \mathrm{~h}$ of degradation.

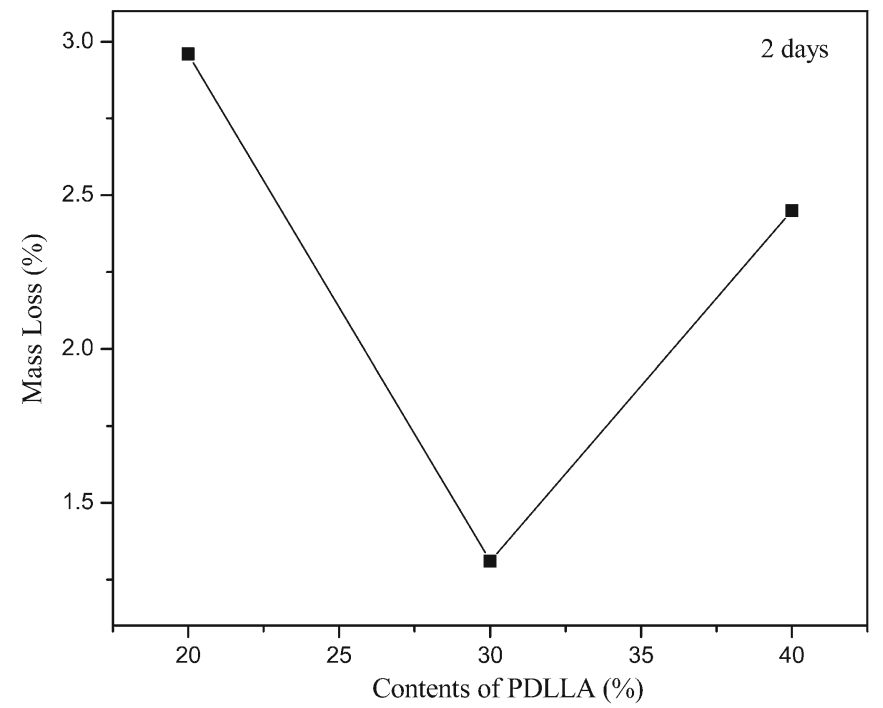

Figure 2. Mass loss of PDLLA/PGA composite films with varying PDLLA contents after 2 days of degradation.

nearly $13 \%$ of its mass. However, when the PDLLA content was $>20 \%$, the composite films underwent minimal mass loss during the degradation process. It was obvious that PDLLA improved the hydrolytic stability of the composite films due to its hydrophobic methyl group in the repeating unit.

Weight loss within this period may be almost entirely attributed to the degradation of PGA because of its high hydrophilicity, and thereby, a high degradation rate. The supplemented PDLLA can protect PGA from rapid degradation by decreasing its hydrophilicity; thus, PGA in composite films undergoes minimal mass loss.

Figure 2 shows changes in the mass loss of PDLLA/PGA composite films with $20 \%, 30 \%$ and $40 \%$ PDLLA after a 2-day degradation period. It is apparent that when the PDLLA content exceeded 30\%, mass loss of the composite films increased instead. This result suggested that when the PDLLA content exceeded 30\%, the phase separation of composite films would be more serious. Therefore, PDLLA cannot protect PGA from water better. Subsequent SEM analysis can confirm that the phenomenon of phase separation occurred in the PDLLA/PGA composite film.

Figure 3 shows changes in the mass loss of different composite films with different PDLGA contents and $30 \%$ PDLLA after 5 days of degradation. The figure shows that when the PDLGA content exceeded $1 \%$, the PDLGA/PDLLA/PGA composite films showed better hydrolytic stability than PDLLA/PGA composite films.

PDLGA as a compatibilizer can act as a bridge between the PDLLA and PGA regions because of the presence of both PDLLA and PGA segments; it can thus optimize the microstructure of the composite films, which can be verified by subsequent SEM micrographs. Consequently, PDLLA can protect PGA from water more effectively. 


\subsection{Morphology}

The degrees of homogeneity and compactness of the blend film could be used to evaluate the compatibility of 2 or more

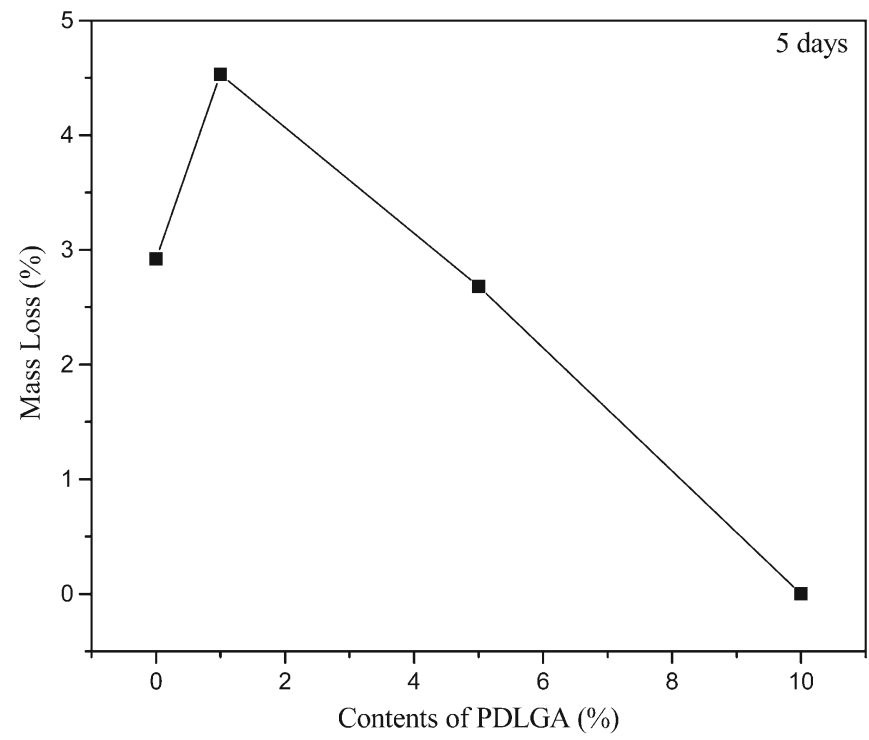

Figure 3. Mass loss of PDLGA/PDLLA/PGA composite films with varying contents of PDLGA and 30\% PDLLA after 5 days of degradation. kinds of polymers. If the blend was homogeneous and compact, different kinds of polymers in this blend would have good miscibility (Ye et al 2006).

In this study, PGA films showed changes in the microstructure after being blended with PDLLA and PDLGA. An SEM analysis provided detailed information on the morphological changes (figure 4). Micrographs of pure PGA film (figures $4 \mathrm{a}, \mathrm{d}$ ) displayed a homogeneous and compact morphology. The morphologies of the $30 \%$ PDLLA/PGA composite film (figures $4 \mathrm{~b}$, e) showed an obvious phase separation because of its disordered and loose microstructure, suggesting a poor miscibility between PDLLA and PGA. But the phase separation decreased after the addition of PDLGA. Micrographs of the 5\% PDLGA $30 \%$ PDLLA/PGA composite film (figures $4 \mathrm{c}, \mathrm{f}$ ) showed a smooth and homogeneous morphology, one that was similar to the pure PGA film, suggesting that PDLGA was effective as a compatibilizer in improving the miscibility of PDLLA and PGA.

\subsection{Thermal analysis}

DSC thermograms of all the composite films (figure 5) showed crystallization thermograms similar to the pure PGA film, but with relatively lower crystallinity (table 1), which is evident from the crystallization peaks. This may have
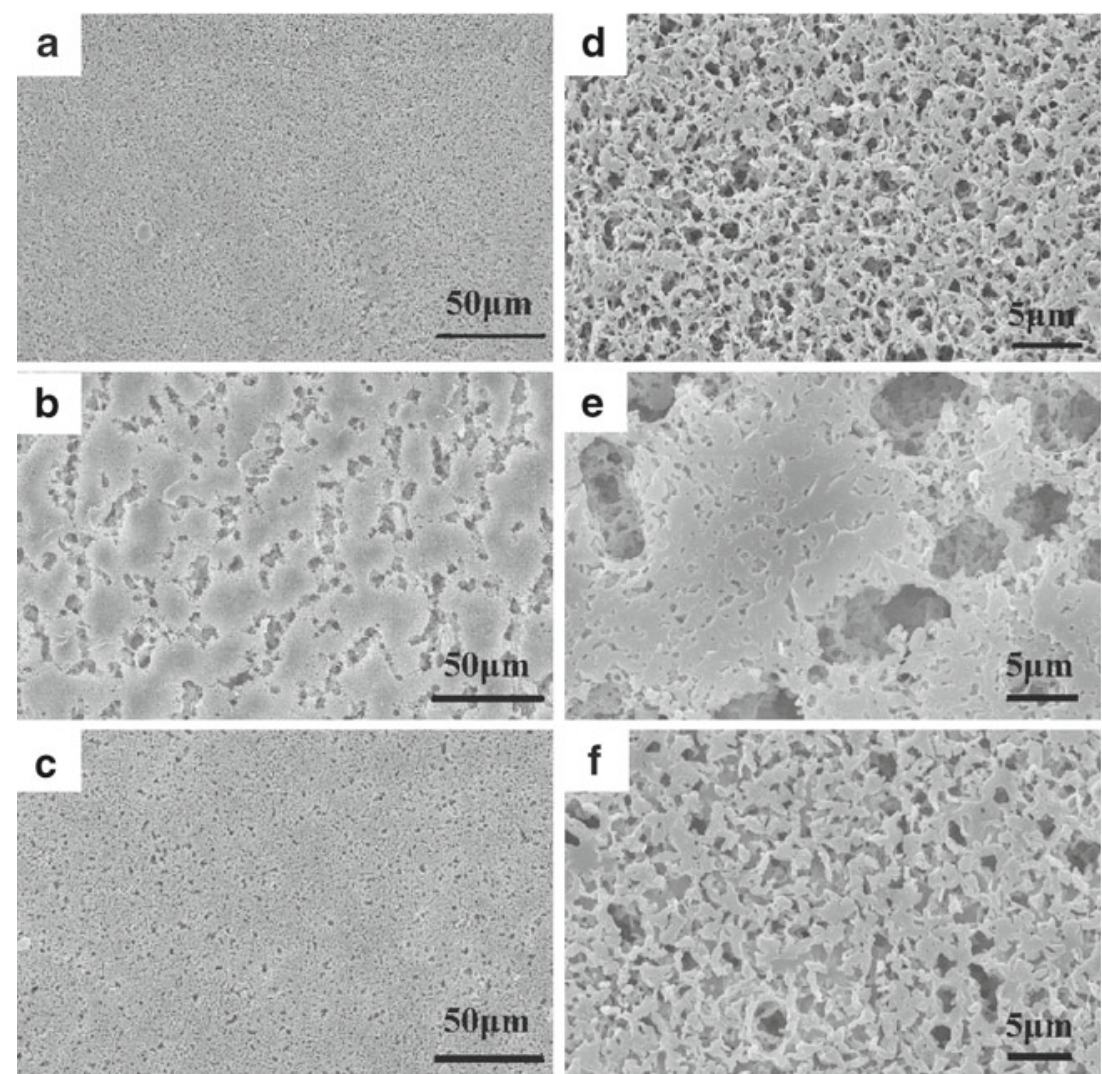

Figure 4. SEM micrographs of pure PGA film (a, d), 30\% PDLLA/PGA film (b, e), and 5\% PDLGA 30\% PDLLA/PGA film (c, f). 


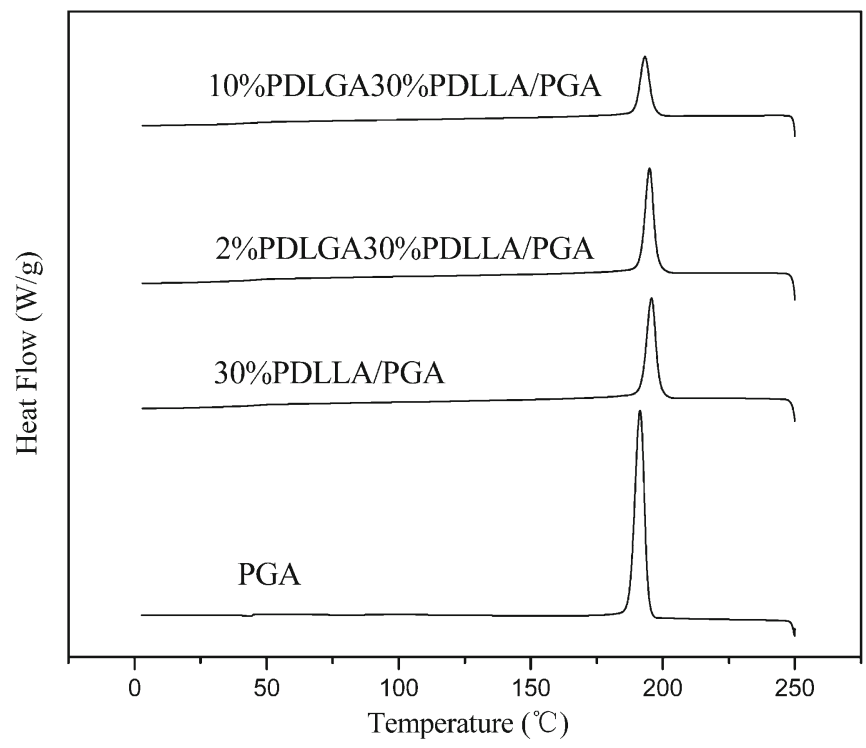

Figure 5. DSC thermograms of PGA film, 30\% PDLLA/PGA film, 2\% PDLGA 30\% PDLLA/PGA film and 10\% PDLGA $30 \%$ PDLLA/PGA film.

Table 1. Crystallinity $\left(x_{\mathrm{c}}, \%\right)$ of PGA and its composite films.

\begin{tabular}{|c|c|c|c|}
\hline \multirow[b]{2}{*}{ Films } & \multicolumn{2}{|c|}{$30 \%$ PDLLA/ $2 \%$ PDLGA/ } & \multirow{2}{*}{$\begin{array}{l}\text { 10\% PDLGA/ } \\
30 \% \text { PDLLA/PGA }\end{array}$} \\
\hline & PGA PGA & $30 \%$ PDLLA/PGA & \\
\hline$x_{\mathrm{c}}(\%)$ & $50 \cdot 9 \quad 38 \cdot 3$ & $36 \cdot 1$ & $31 \cdot 5$ \\
\hline
\end{tabular}

occurred because of the presence of PDLGA and PDLLA, which might have hindered PGA crystallization.

PGA is a crystalline polymer with a high degree of crystallinity but PDLGA and PDLLA are amorphous macromolecules (Zong et al 2003) and their segments are not as compact. After being blended with PDLGA and PDLLA, the orderly arrangement of the PGA segments is disrupted to a certain extent, indicating that the perfect crystal structure in PGA would be slightly disrupted, with a decrease in the crystallinity.

\section{Conclusions}

A series of PDLLA/PGA and PDLGA/PDLLA/PGA composite films were prepared by solution casting, using HFIP as the solvent. All the films were degraded in vitro in the order of PGA $>10 \%$ PDLLA/PGA $>20 \%$ PDLLA/PGA > $40 \%$ PDLLA/PGA $>1 \%$ PDLGA $30 \%$ PDLLA/PGA $>30 \%$ PDLLA/PGA $>2 \%$ PDLGA $30 \%$ PDLLA/PGA $>5 \%$ PDLGA 30\% PDLLA/PGA > 10\% PDLGA 30\% PDLLA/ PGA. A smooth and homogeneous morphology of 5\% PDLGA 30\% PDLLA/PGA, which was similar to the pure PGA film, suggested that PDLGA was effective as a compatibilizer in improving the miscibility of PDLLA and PGA. In all the films, the optimum ratio of PDLGA, PDLLA and PGA for obtaining a smooth and homogeneous morphology was $5 \%, 30 \%$ and $65 \%$, respectively.

In conclusion, PDLGA as a compatibilizer improved the compatibility of PDLLA/PGA composite films, thereby increasing the hydrolytic stability of the PDLLA/PGA composite films. We expected that this study will show some direction in modifying the degradation of PGA.

\section{Acknowledgement}

The authors would like to thank Hubei Key Laboratory of Mine Environmental Pollution Control and Remediation for support.

\section{References}

Choueka J et al 1995 J. Biomed. Mater. Res. 291309

Doi Y, Steinbuchel A and Chen G Q 2004 Biopolymers polyesters III: applications and commercial products (Beijing: Chemical Industry Press) Vol. 4, p. 152 [in Chinese]

Montes de Oca H and Ward IM 2006 Polymer 477070

Ye X, Kennedy J F, Li B and Xie B J 2006 Carbohyd. Polym. 64532

You Y, Lee S W, Youk J H, Min B-M, Lee S J and Park W H 2005 Polym. Degrad. Stabil. 90441

Zong X H, Ran S F, Kim K-S, Fang D F, Hsiao B S and Chu B 2003 Biomacromolecules 4416 